\title{
Tetrahydrocurcumin protects against spinal cord injury and inhibits the oxidative stress response by regulating FOXO4 in model rats
}

\author{
JIANCHENG XI, XIAOBO LUO, YIPENG WANG, JINGLONG LI, LIXIN GUO, \\ GUANGSENG WU and QINGUI LI \\ Department of Minimally Invasive Spine Surgery, \\ The 309th Hospital of The People's Liberation Army, Beijing 100091, P.R. China
}

Received March 5, 2017; Accepted April 6, 2018

DOI: $10.3892 /$ etm.2019.7974

\begin{abstract}
It has been reported that tetrahydrocurcumin has hypoglycemic, hypolipidemic, anti-metastasis, anticancer and anti-depressant pharmacological effects, and its antioxidative, hypoglycemic and hypolipidemic properties are better than those of curcumin. The present study assessed whether tetrahydrocurcumin exerts a neuroprotective effect against spinal cord injury (SCI) and investigated the underlying mechanisms. In a rat model of SCI, tetrahydrocurcumin enhanced the average Basso-Beattie-Bresnahan scores, inhibited water accumulation in the spinal cord and decreased inflammatory factors. Furthermore, oxidative stress and apoptosis (caspase-3 activity and B-cell lymphoma 2-associated X protein levels) were also suppressed in SCI rats treated with tetrahydrocurcumin. Tetrahydrocurcumin effectively decreased the gene expression of matrix metalloproteinase-3 and -13 , as well as cyclooxygenase-2, promoted the phosphorylation of Akt and enhanced the protein expression of forkhead box (FOX)O4 in SCI rats. The present study delineates that tetrahydrocurcumin protects against SCI and inhibits the oxidative stress response by regulating the $\mathrm{FOXO} 4$ in SCI model rats.
\end{abstract}

\section{Introduction}

In spinal cord injury (SCI), in addition to direct injury caused by primary trauma, the secondary pathological changes continue to expand around it; these include ischemia, free radical damage, inflammatory response, excitotoxicity, neuronal degeneration and necrosis, as well as apoptosis (1).

Correspondence to: Mr. Qingui Li, Department of Minimally Invasive Spine Surgery, The 309th Hospital of The People's Liberation Army, 17 Montenegro Jhao Road, Beijing 100091, P.R. China

E-mail: qxpc52752927@126.com

Key words: tetrahydrocurcumin, spinal cord injury, oxidative stress, forkhead box $\mathrm{O}$
Lipid peroxidation is one of the earliest biochemical changes after SCI, which is a continuation of free radical damage, and it is considered to be an important factor leading to the exacerbation of the original injury area. The inflammatory response acts as double-edged sword in SCI-inflammatory cells are extensively accumulated in the early stage, and secrete a large number of toxic cytokines and free radicals so as to aggravate SCI, while protective cytokines are synthesized in the late stage $(2,3)$.

SCI may arise from high falls and traffic accidents, and is common in the clinic (4). After SCI, primary injury and secondary injury (including apoptosis) of spinal cord neurons are the main causes of spinal nerve dysfunction, but the secondary injury of neurons may have more serious consequences (5). After injury, the neuron has a relatively low capacity to undergo self-repair and regeneration, the regulation of which requires the participation of numerous genes, including nerve growth factor and apoptosis-inhibiting genes (6). The initiation of these genes requires signal transduction through the associated pathways (3).

Forkhead box (FOX)O transcription factors are regulated by a variety of external stimuli, including insulin, insulin-like growth factor I, nutritional status, cytokines and stress (7). These external factors regulate the subcellular localization, DNA binding properties, protein levels and transcriptional activity of FOXO through the complex combination of modification and translation of FOXO, including phosphorylation, acetylation and methylation (8). FOXO has been proved to be involved in protein degradation and synthesis, which also take part in the regulation of skeletal muscle growth and development (9).

Tetrahydrocurcumin (Fig. 1), the most active and major metabolite of curcumin in vivo, may be isolated from the cytoplasm of the small intestine and liver after the administration of curcumin to humans or mice (10). It inhibits tyrosinase and the formation of oxygen free radicals, and removes already formed free radicals, thereby exerting a significant antioxidant effect; it has therefore been employed as a natural functional whitening raw material for cosmetic research and development (11). Tetrahydrocurcumin has a pharmacological effect similar to that of curcumin (11). To date, tetrahydrocurcumin 
has been reported to have various pharmacological activities, including anti-metastasis, anticancer, antioxidant, free radical scavenging, hypoglycemic and hypolipidemic effects $(12,13)$. The present study assessed the potential neuroprotective effects of tetrahydrocurcumin in a rat model of SCI and investigated the underlying mechanisms.

\section{Materials and methods}

Animals and establishment of the SCI model. Male Sprague Dawley rats (weight, 180-230 g; age, 8-10 weeks) were purchased from Beijing Vital River Laboratory Animal Technology Co., Ltd (Beijing, China) and kept under standard housing conditions (temperature, $22-23^{\circ} \mathrm{C}$; humidity, $55-65 \%$; 12 -h light/dark cycle). Food and water were provided ad libitum. The rats were randomly assigned to one of three groups ( $\mathrm{n}=8$ in each): Sham-control group, SCI group and tetrahydrocurcumin treatment group. In brief, the rats were anesthetized with sodium pentobarbital ( $30 \mathrm{mg} / \mathrm{kg}$ body weight, i.p.). For the SCI and tetrahydrocurcumin treatment groups, a dorsal laminectomy was performed at the 8th thoracic vertebra level to expose the spinal cord. Subsequently, T6 and T10 were clamped to secure the vertebral column and a hammer was dropped to produce a moderate SCI model. Following aneasthetisation, rats in the sham-control group were treated with $100 \mu \mathrm{l}$ normal saline. Following SCI and wound suturing, the injured rats were treated with $100 \mu 1$ normal saline or tetrahydrocurcumin $(80 \mathrm{mg} / \mathrm{kg} / \mathrm{day}, 2$ weeks, i.p.) in the SCI and tetrahydrocurcumin treatment groups, respectively. All animal experiments were approved by the Ethics Committee of The 309th Hospital of The People's Liberation Army (Beijing, China).

Analysis of rat behavior and water accumulation. After treatment with tetrahydrocurcumin, the motor function of the rats was evaluated according to the Basso, Beattie and Bresnahan (BBB) scale (0-21 points) at week 1 and 2. Rats were allowed to freely walk around for $4 \mathrm{~min}$ in an open field, and the movements of the hindlimbs were observed and scored (14). Then, the spinal cord tissue was collected and weighed; this was termed the wet weight. Spinal cord tissue was dried at $80^{\circ} \mathrm{C}$ for $48 \mathrm{~h}$ and weighed again; this was term the dry weight. Water accumulation was calculated as (dry weight/wet weight) x100\%.

Analysis of inflammation and oxidative stress. Following tetrahydrocurcumin treatment whole blood was collected and centrifugation at $2,000 \mathrm{x} \mathrm{g}$ at $4^{\circ} \mathrm{C}$ for $10 \mathrm{~min}$ to obtain serum, the serum was then stored at $-80^{\circ} \mathrm{C}$ for analysis. (NF)- $\mathrm{kB}$ p65 (cat. no. H202), tumor necrosis factor (TNF)- $\alpha$ (cat. no. H052), interleukin (IL)-1ß (cat. no. H002), IL-6 (cat. no. H007) and malondialdehyde (MDA; cat. no. A003-1) levels, as well as superoxide dismutase (SOD; cat. no. A001-1-1), glutathione (GSH; cat. no. A006-2) and GSH peroxidase (GSH-PX; cat. no. A005) activity were measured using ELISA kits (Nanjing Jiancheng Biology Engineering Institute, Nanjing, China).

Western blot analysis. Spinal cord tissue samples were placed in ice-cold saline and homogenized with

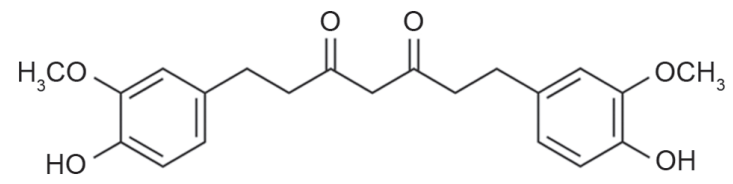

Figure 1. Chemical structure of tetrahydrocurcumin.

radioimmunoprecipitation assay buffer for $30 \mathrm{~min}$. The supernatant was collected after centrifugation at $15,000 \mathrm{xg}$ for $10 \mathrm{~min}$ at $4^{\circ} \mathrm{C}$ and the protein concentration was determined using a BSA assay (Bio-Rad Laboratories, Inc., Hercules, CA, USA). Protein (50 $\mu \mathrm{g} /$ lane) was loaded onto a $10 \%$ SDS-PAGE gel for electrophoresis, and then transferred onto a polyvinylidene difluoride membrane (EMD Millipore, Billerica, MA, USA). The membrane was blocked with $5 \%$ non-fat powdered milk in Tris-buffered saline containing Tween-20 (TBST) for $1 \mathrm{~h}$ at $37^{\circ} \mathrm{C}$ and incubated with antibodies to B-cell lymphoma 2 (Bcl-2)-associated $\mathrm{X}$ protein (Bax; cat. no. sc-6236; 1:1,000), matrix metalloproteinase (MMP)-3 (cat. no. 14351; 1:1,000; Cell Signaling Technology, Inc., Danvers, MA, USA), MMP-13 (cat. no. sc-30073; 1:1,000), cyclooxygenase (COX)-2 (cat. no. sc-7951; 1:1,000), phosphorylated (p)-Akt (cat. no. sc-7985-R; 1:2,000), FOXO4 (cat. no. sc-373877; 1:1,000) and GAPDH (cat. no. sc-25778; 1:5,000; all Santa Cruz Biotechnology, Inc., Dallas, TX, USA) overnight at $4^{\circ} \mathrm{C}$. The membrane was washed with TBST and then incubated with horseradish peroxidase-conjugated goat anti-mouse or anti-rabbit immunoglobulin G (cat. nos. sc-2005 and sc-2004, respectively; 1:5,000; Santa Cruz Biotechnology, Inc.) as the secondary antibody at room temperature for $1 \mathrm{~h}$. Protein bands was observed using BeyoECL Moon (Beyotime Institute of Biotechnology, Haimen, China) and analyzed using Image $\mathrm{Lab}^{\mathrm{TM}}$ software (version 3.0; Bio-Rad Laboratories, Inc.).

Caspase-3 activity. Spinal cord tissue samples were placed in ice-cold saline and homogenized with radioimmunoprecipitation assay buffer for $30 \mathrm{~min}$. The supernatant was collected after centrifugation at $15,000 \mathrm{x} g$ for $10 \mathrm{~min}$ at $4^{\circ} \mathrm{C}$ and the protein concentration was determined using a Bio-Rad kit (Bio-Rad Laboratories, Inc., Hercules, CA, USA). Protein $(10 \mu \mathrm{g})$ was incubated with a caspase-3 activity kit (cat. no. C1116; Beyotime Institute of Biotechnology). Caspase-3 activity was measured in a spectrophotometer at $405 \mathrm{~nm}$.

Statistical analysis. Values are expressed as the mean \pm standard deviation using SPSS software (version 17.0; SPSS, Inc., Chicago, IL, USA). Data were analyzed using one-way analysis of variance followed by Dunnett's post-hoc test. $\mathrm{P}<0.05$ was considered to indicate a statistically significant difference.

\section{Results}

Tetrahydrocurcumin enhances $B B B$ scores and reduces the water content in the spinal cord of SCI rats. Compared with the control group, a significant reduction in BBB scores at all time points and an increase in the water content in the spinal 
A

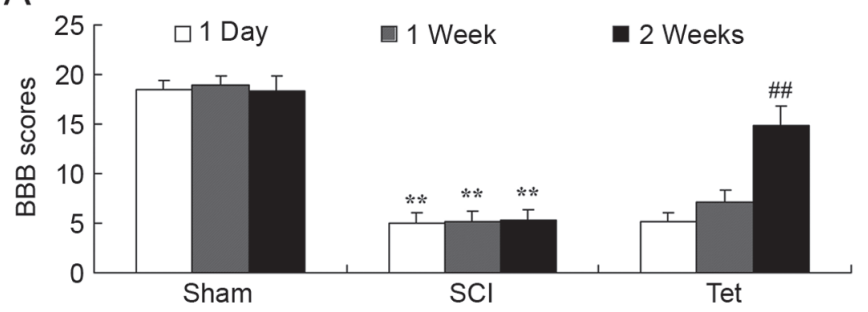

B

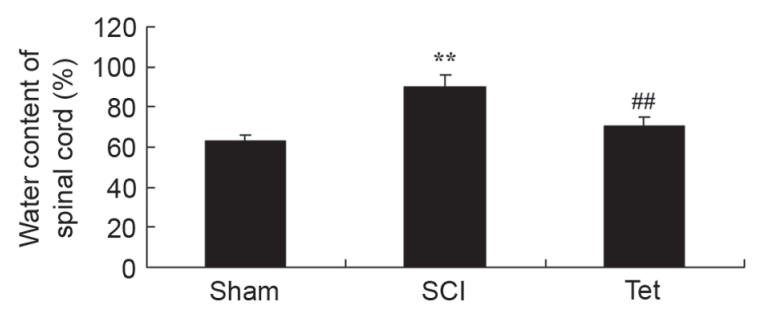

Figure 2. Tetrahydrocurcumin enhances BBB scores and inhibits water content of spinal cord in SCI rats. (A) BBB scores and (B) water content of the spinal cord. ${ }^{* *} \mathrm{P}<0.01$ compared with sham control group, ${ }^{\# \#} \mathrm{P}<0.01$ compared with SCI model group. Groups: Sham, sham control group; SCI, SCI model group; Tet, tetrahydrocurcumin treatment group; SCI, spinal cord injury; BBB, Basso-Beattie-Bresnahan.

A

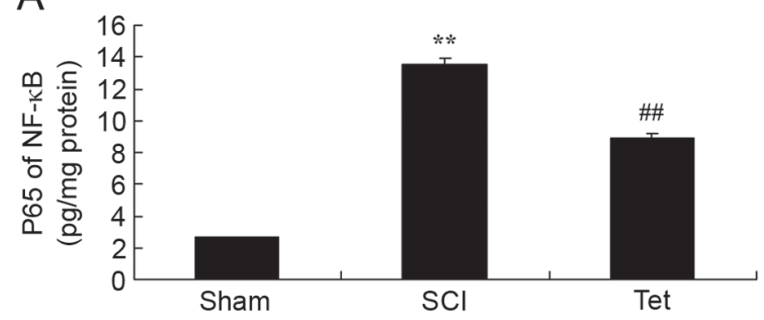

C

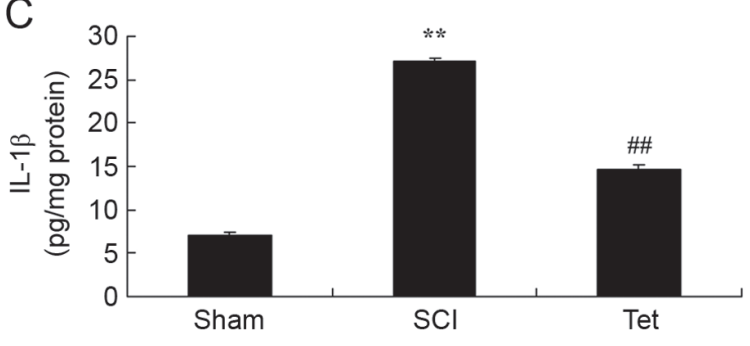

B
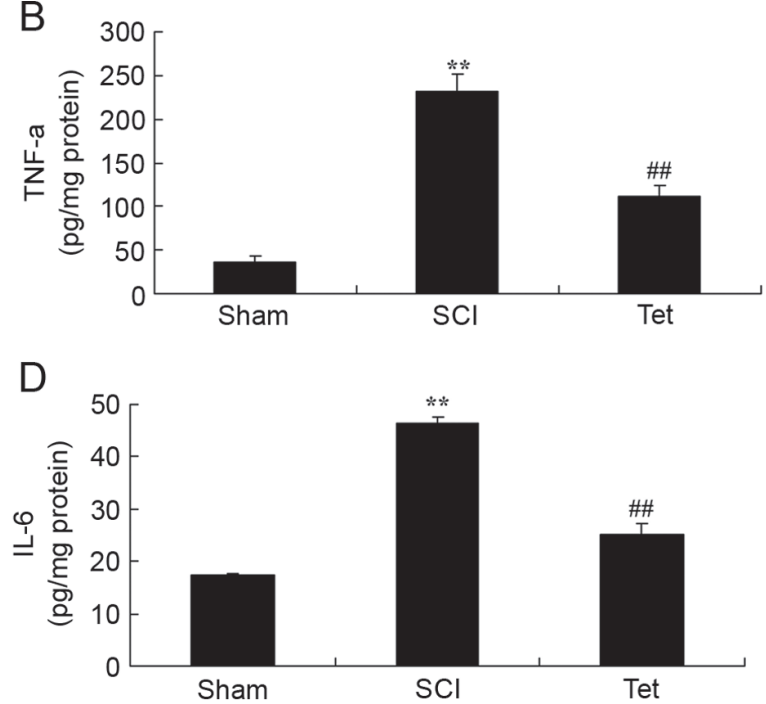

Figure 3. Tetrahydrocurcumin inhibits inflammation in SCI rats. Tetrahydrocurcumin inhibits (A) NF- $\kappa$ B p65, (B) TNF- $\alpha,(\mathrm{C}) \mathrm{IL}-1 \beta$ and (D) IL-6 in SCI rats. ${ }^{* *} \mathrm{P}<0.01$ compared with sham control group, ${ }^{\#} \mathrm{P}<0.01$ compared with SCI model group. Groups: Sham, sham control group; SCI, SCI model group; Tet, tetrahydrocurcumin treatment group; SCI, spinal cord injury; NF, nuclear factor; TNF, tumor necrosis factor; IL, interleukin.

cord was observed in SCI rats (Fig. 2). Administration of tetrahydrocurcumin to SCI rats resulted in a significant increase of BBB scores at week 2 and inhibition of water accumulation in the spinal cord compared with that in the SCI model group (Fig. 2).

Tetrahydrocurcumin inhibits inflammation in SCI rats. ELISAs indicated that the serum levels of p65 of $\mathrm{NF}-\kappa \mathrm{B}$, TNF- $\alpha$, IL-1 $\beta$ and IL- 6 levels were notably enhanced in the SCI model group compared with those in the control group (Fig. 3). Treatment of SCI rats with tetrahydrocurcumin significantly inhibited p65 of NF- $\kappa \mathrm{B}, \mathrm{TNF}-\alpha$, IL-1 $\beta$ and IL-6 levels (Fig. 3).

Tetrahydrocurcumin inhibits oxidative stress in SCI rats. As presented in Fig. 4, overproduction of MDA, as well as inhibition of SOD, GSH and GSH-PX activity were observed in SCI rats compared with the control group (Fig. 4). Administration of tetrahydrocurcumin to SCI rats significantly decreased MDA levels, and promoted the activity of SOD, GSH and GSH-PX (Fig. 4).

Tetrahydrocurcumin inhibits apoptosis in SCI rats. As indicated in Fig. 5, caspase-3 activity and Bax protein expression were significantly enhanced in the SCI model group compared with those in the control group. However, treatment of SCI rats with tetrahydrocurcumin significantly suppressed caspase-3 activity and Bax protein expression (Fig. 5).

Tetrahydrocurcumin inhibits the protein expression of $M M P-3, M M P-13$ and COX-2 in SCI rats. To evaluate the mechanisms by which tetrahydrocurcumin attenuates SCI, MMP-3, MMP-13 and COX-2 expression were measured in the rats of the different experimental groups. As presented in Fig. 6, MMP-3, MMP-13 and COX-2 expression in SCI rats were higher than those in the control group. Treatment with tetrahydrocurcumin suppressed the protein expression of MMP-3, MMP-13 and COX-2 in SCI rats.

Tetrahydrocurcumin enhances the protein levels of $p$-Akt and FOXO4 in SCI rats. To further evaluate the mechanism by which tetrahydrocurcumin attenuates SCI, p-Akt levels and FOXO4 expression were measured in the rats of the different experimental groups. p-Akt levels and FOXO4 expression in SCI rats were markedly lower than those in the control group (Fig. 7). However, tetrahydrocurcumin induced the production of p-Akt and the protein expression of FOXO4 in SCI rats (Fig. 7). 
A

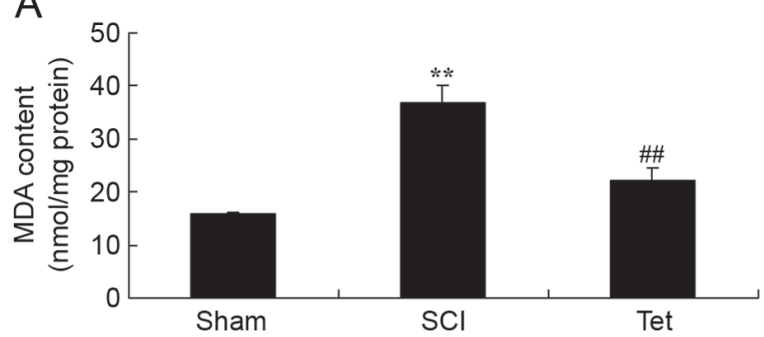

C

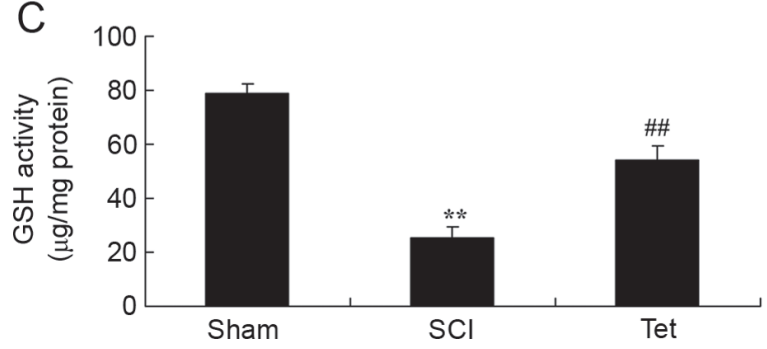

B

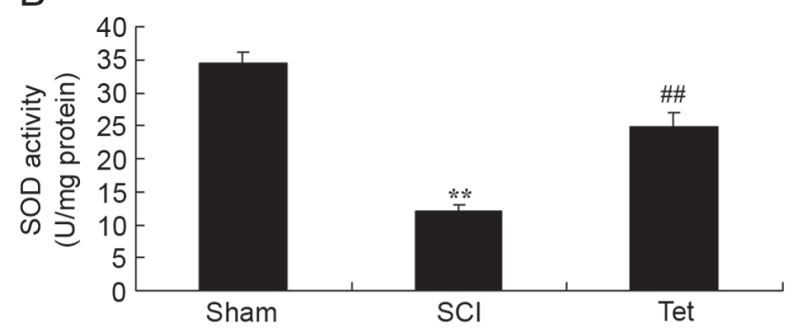

D

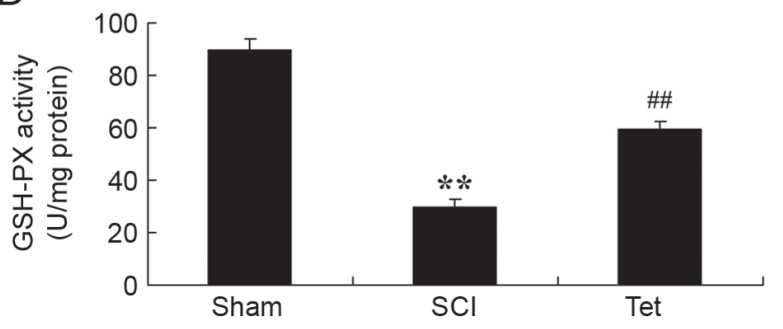

Figure 4. Tetrahydrocurcumin inhibits oxidative stress in SCI rats. Tetrahydrocurcumin inhibits (A) MDA, (B) SOD, (C) GSH and (D) GSH-PX in SCI rats. ${ }^{* *} \mathrm{P}<0.01$ compared with sham control group, ${ }^{\# \prime} \mathrm{P}<0.01$ compared with SCI model group. Groups: Sham, sham control group; SCI, SCI model group; Tet, tetrahydrocurcumin treatment group; SCI, spinal cord injury; MDA, malondialdehyde; SOD, superoxide dismutase; GSH, glutathione; GSH-PX, GSH peroxidase.
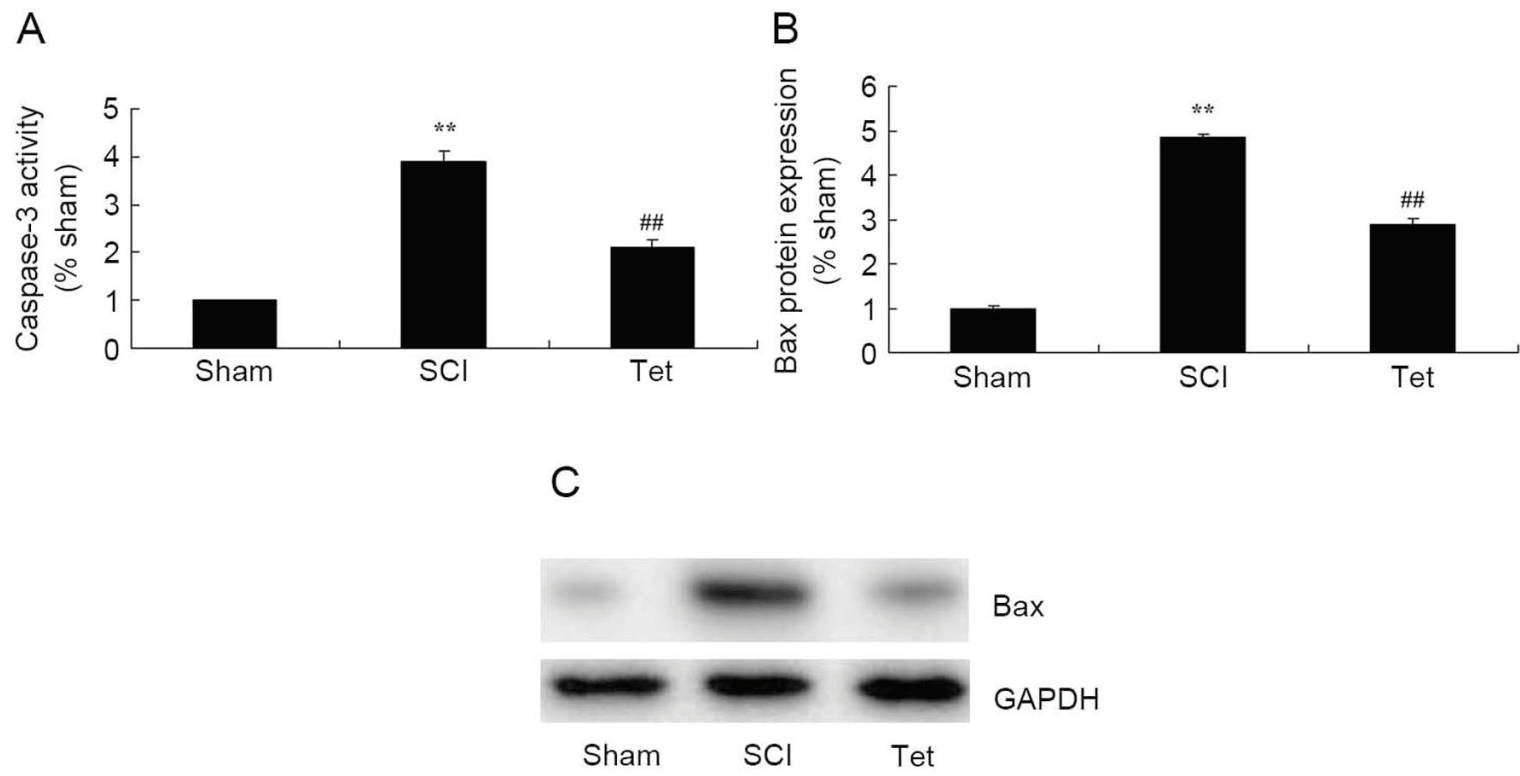

Figure 5. Tetrahydrocurcumin inhibits apoptosis in SCI rats. (A) Caspase-3 activity. (B and C) Bax protein expression detected by western blot analysis.

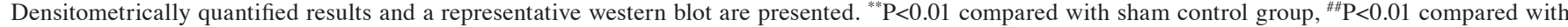
SCI model group. Groups: Sham, sham control group; SCI, SCI model group; Tet, tetrahydrocurcumin treatment group; SCI, spinal cord injury; Bax, B-cell lymphoma 2-associated X protein.

\section{Discussion}

SCI is a serious injury of the central nervous system and severely impairs the quality of life of affected patients (15). Although the survival rate and survival time of SCI patients has significantly increased with the development of modern medicine, most patients affected are disabled for life due to the difficulty of neuronal regeneration (16). SCI represents a global medical challenge, and a vast amount of scientific and clinical research has achieved significant progress in the field (17). In particular, a large number of clinical studies and animal experiments have demonstrated that a series of changes in molecular signaling and pathological processes occur internally after SCI, including neuronal apoptosis, inflammatory response and axonal demyelination (18). The results of the present study preliminarily confirm that tetrahydrocurcumin enhances BBB scores, inhibits water accumulation in the spinal cord, and decreases inflammatory factors, oxidative stress and apoptosis in SCI rats. Sangartit et al (10) reported that tetrahydrocurcumin protects against cadmium-induced hypertension in mice through exerting antioxidative and anti-inflammatory effects.

MMPs have an important role in regulating the development of the central nervous system $(19,20)$. However, in the 
A

듬

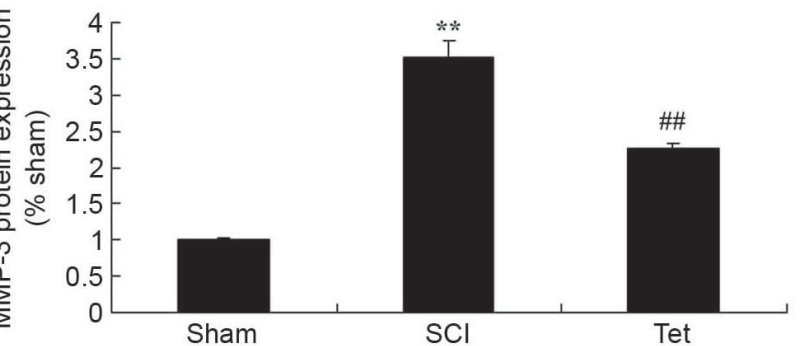

C

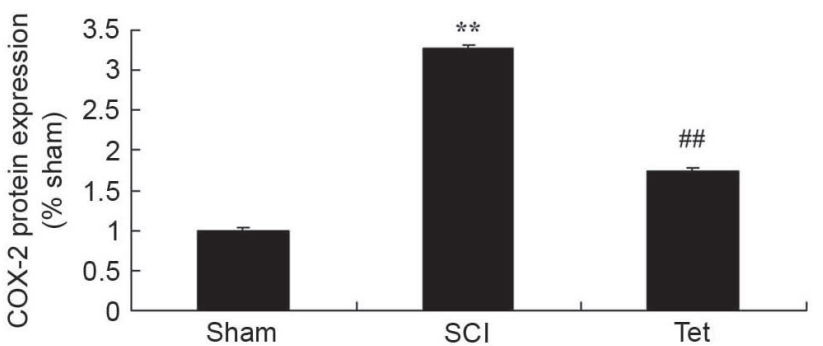

$\mathrm{B}$

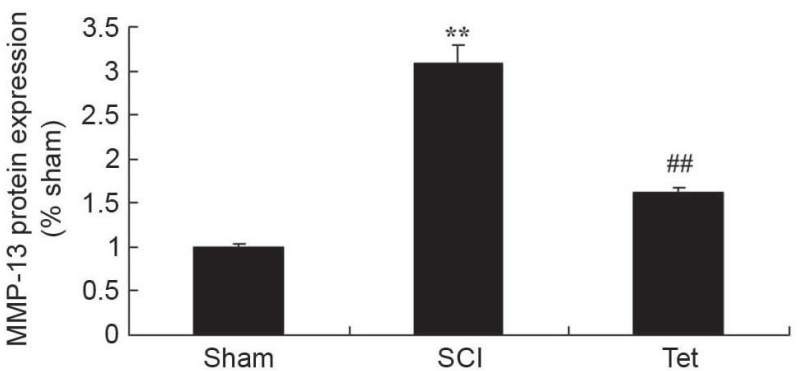

$\mathrm{D}$

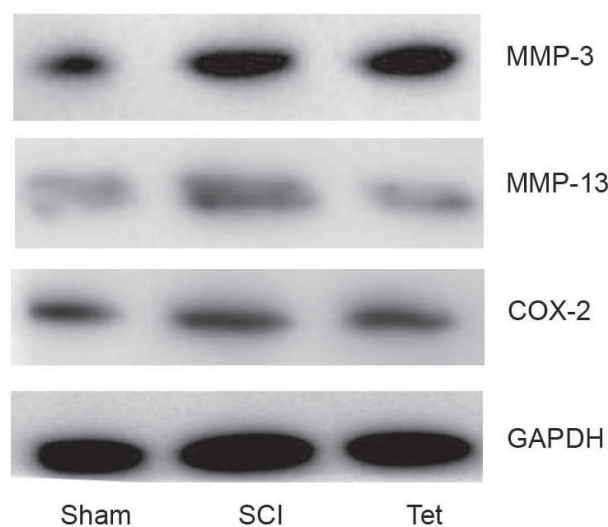

Figure 6. Tetrahydrocurcumin inhibits the protein expression of MMP-3, MMP-13 and COX-2 in SCI rats. Western blot analysis was used to determine the protein levels of (A) MMP-3, (B) MMP-13 and (C) COX-2 (densitometric quantification results). (D) Representative western blot image. ${ }^{* *} \mathrm{P}<0.01$ compared with sham control group, ${ }^{\# \#} \mathrm{P}<0.01$ compared with SCI model group. Groups: Sham, sham control group; SCI, SCI model group; Tet, tetrahydrocurcumin treatment group; SCI, spinal cord injury; MMP, matrix metalloproteinase; COX, cyclooxygenase.
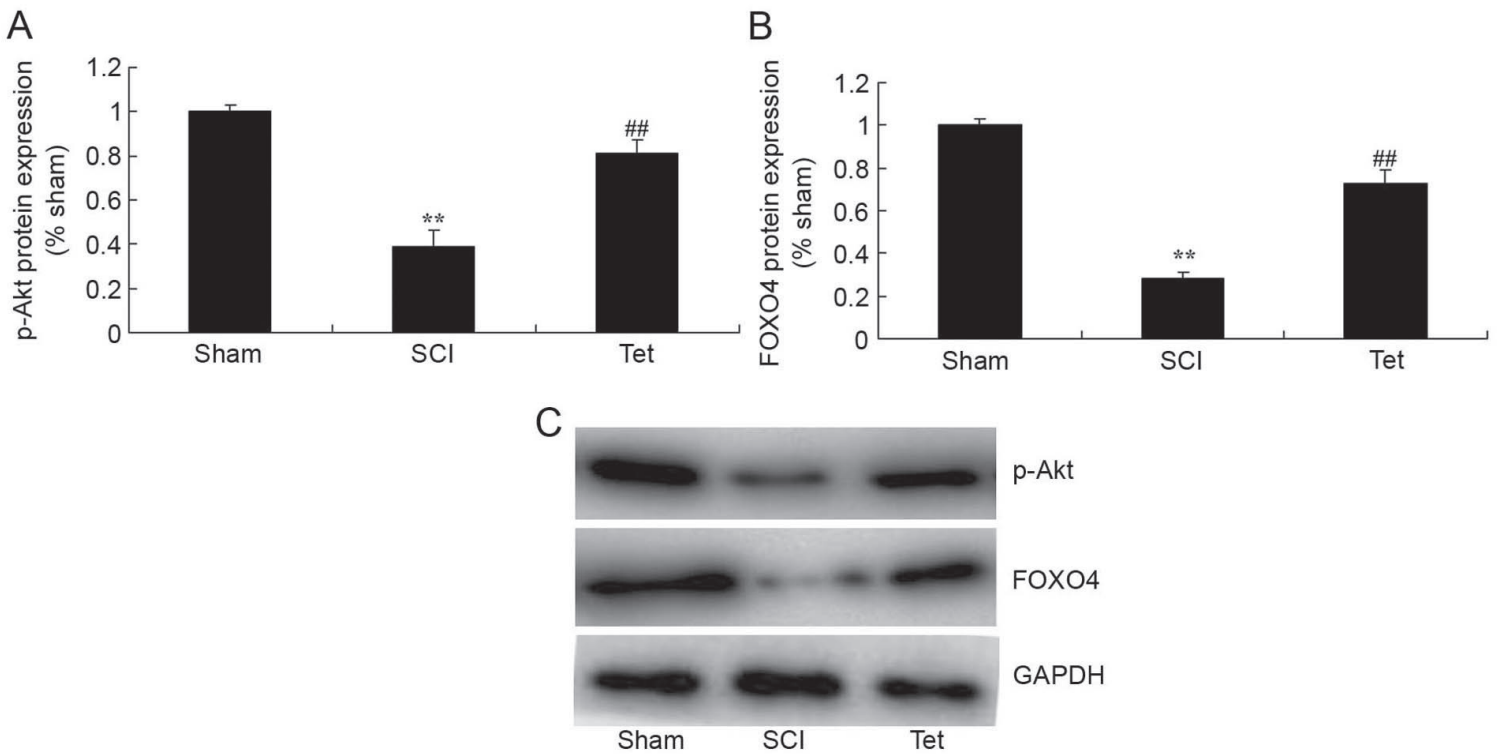

Figure 7. Tetrahydrocurcumin induces p-Akt and expression of FOXO4 in SCI rats. Western blot analysis was used to determine the protein levels of (A) p-Akt and (B) FOXO4 (densitometric quantification results). (C) Representative western blot image. ${ }^{* * *} \mathrm{P}<0.01$ compared with sham control group, ${ }^{\# \#} \mathrm{P}<0.01$ compared with SCI model group. Groups: Sham, sham control group; SCI, SCI model group; Tet, tetrahydrocurcumin treatment group; SCI, spinal cord injury; p-Akt, phosphorylated Akt; FOXO, forkhead box O.

presence of neurological disorders, MMP members exhibit an abnormally increased expression. In recent years, a large number of basic studies indicated that MMPs are involved in various pathological processes after SCI (21). The extracellular matrix $(\mathrm{ECM})$ is composed of a variety of proteins and non-proteins, which make up the microenvironment for cells and exert supporting, connecting, nutritional and defense functions (22). The basement membrane of blood vessels and the ECM are important components maintaining blood-spinal cord barrier integrity (23). MMPs may cause the damage to 
the blood-spinal cord barrier by degrading ECM components, leading to increased permeability and extravasation of capillary water and plasma protein, resulting in the increases in the water content in the intracellular clearance and the formation of spinal cord edema (24). It was also demonstrated that MMPs have an important role in the inflammatory response and apoptosis after SCI (20). The results of the present study indicate that treatment with tetrahydrocurcumin suppressed the protein expression of MMP-3, MMP-13 and COX-2 in SCI rats. Yodkeeree et al (25) revealed that tetrahydrocurcumin inhibits the migration and invasion of HT1080 cells through MMPs and urokinase-type plasminogen activator.

FOXOs exert their effects as transcription factors through direct binding with target genes and interactions with other transcriptional regulators, and their presence in cells affects their function, including cell cycle regulation, apoptosis and cellular metabolism $(7,26)$. It has been demonstrated that FOXO is involved in the degradation and synthesis of protein in skeletal muscle, which maintains the protein content of skeletal muscle via interaction with phosphoinositide-3 kinase (PI3K), mammalian target of rapamycin (mTOR) complex 1 (mTORC1) and NF- $\mathrm{kB}$ in the face of external stimuli (27). Study of the molecular mechanisms of FOXO and its associated signaling pathways in the regulation of muscle degradation and synthesis may provide novel approaches for maintaining the normal development of skeletal muscle (28). The present study confirmed that tetrahydrocurcumin induces FOXO4 expression in SCI rats, which was decreased after SCI. Xiang et al (13) reported that tetrahydrocurcumin inhibits the oxidative stress response through FOXO forkhead transcription factor.

The PI3K/Akt/mTOR signal transduction pathway is an important pathway for receptor signal transduction to the cell, which may regulate cell differentiation and proliferation, as well as inhibit apoptosis (19). Under normal physiological conditions, intracellular expression of PI3K tends to be low (19). When the cells are damaged its expression rapidly increases, leading to the phosphorylation of phosphatidylinositol to generate phosphatidylinositol 3,4-bisphosphate, the latter of which may phosphorylate Akt to generate p-Akt, which in turn phosphorylates mTOR at the Ser2448 site to thereby activate it (28). The PI3K/Akt signaling pathway is an important signal transduction pathway inside cells, which is closely associated with numerous vital cellular activities (29). Phosphorylation of Akt may promote cell survival by inhibiting glycogen synthase kinase, tumor suppressors and caspase- 3 phosphorylation; following phosphorylation, Akt then dissociates from the Bcl-2 and 14-3-3 receptor protein complex, resulting in an anti-apoptotic effect (30). The present study demonstrated that tetrahydrocurcumin reduces the SCI-associated inhibition of p-Akt levels and FOXO4 expression in rats. Wu et al (12) demonstrated that tetrahydrocurcumin induces autophagic cell death of human leukemia HL-60 cells through coordinative modulation of $\mathrm{PI} 3 \mathrm{~K} / \mathrm{Akt} / \mathrm{mTOR}$ signaling pathways.

In conclusion, the present study indicated that tetrahydrocurcumin improves BBB scores and inhibits the oxidative stress response by regulating the FOXO4 in SCI model rats. Therefore, tetrahydrocurcumin may be applied as one of the clinical adjunctive therapies for SCI. The present study should be followed by further clinical studies.

\section{Acknowledgements}

Not applicable.

\section{Funding}

No funding was received.

\section{Availability of data and materials}

The analyzed data sets generated during the study are available from the corresponding author on reasonable request.

\section{Authors' contributions}

JX designed the experiment and wrote the manuscript. XL, YW, JL, LG, GW and QL performed the experiments. JX and $\mathrm{XL}$ analyzed the data.

\section{Ethics approval and consent to participate}

All animal experiments were approved by the Ethics Committee of The 309th Hospital of The People's Liberation Army.

\section{Patient consent for publication}

Not applicable.

\section{Competing interests}

The authors declare that they have no competing interests.

\section{References}

1. Jiang W, Huang Y, He F, Liu J, Li M, Sun T, Ren W, Hou J and Zhu L: Dopamine D1 receptor agonist A-68930 inhibits NLRP3 inflammasome activation, controls inflammation, and alleviates histopathology in a rat model of spinal cord injury. Spine (Phila Pa 1976) 41: E330-E334, 2016.

2. Hossain MS, Harvey LA, Rahman MA, Muldoon S, Bowden JL, Islam MS, Jan S, Taylor V, Cameron ID, Chhabra HS, et al: Community-based InterVentions to prevent serIous Complications (CIVIC) following spinal cord injury in Bangladesh: Protocol of a randomised controlled trial. BMJ Open 6: e010350, 2016

3. Jan YK and Crane BA: Wheelchair tilt-in-space and recline does not reduce sacral skin perfusion as changing from the upright to the tilted and reclined position in people with spinal cord injury. Arch Phys Med Rehabil 94: 1207-1210, 2013.

4. Triolo RJ, Bailey SN, Miller ME, Rohde LM, Anderson JS, Davis JA Jr, Abbas JJ, DiPonio LA, Forrest GP, Gater DR Jr and Yang LJ: Longitudinal performance of a surgically implanted neuroprosthesis for lower-extremity exercise, standing, and transfers after spinal cord injury. Arch Phys Med Rehabil 93: 896-904, 2012

5. Kressler J, Nash MS, Burns PA and Field-Fote EC: Metabolic responses to 4 different body weight-supported locomotor training approaches in persons with incomplete spinal cord injury. Arch Phys Med Rehabil 94: 1436-1442, 2013.

6. Wang S, Lu J, Li YA, Zhou H, Ni WF, Zhang XL, Zhu SP, Chen BB, Xu H, Wang XY, et al: Autologous olfactory lamina propria transplantation for chronic spinal cord injury: Three-year follow-up outcomes from a prospective double-blinded clinical trial. Cell Transplant 25: 141-157, 2016.

7. Xia M and Zhu Y: FOXO3a involvement in the release of TNF- $\alpha$ stimulated by ATP in spinal cord astrocytes. J Mol Neurosci 51: 792-804, 2013. 
8. Zhang S, Huan W, Wei H, Shi J, Fan J, Zhao J, Shen A and Teng H: FOXO3a/p27kipl expression and essential role after acute spinal cord injury in adult rat. J Cell Biochem 114: 354-365, 2013.

9. Léger B, Senese R, Al-Khodairy AW, Dériaz O, Gobelet C, Giacobino JP and Russell AP: Atrogin-1, MuRF1, and FoXO, as well as phosphorylated GSK-3beta and 4E-BP1 are reduced in skeletal muscle of chronic spinal cord-injured patients. Muscle Nerve 40: 69-78, 2009.

10. Sangartit W, Kukongviriyapan U, Donpunha W, Pakdeechote P, Kukongviriyapan V, Surawattanawan P and Greenwald SE: Tetrahydrocurcumin protects against cadmium-induced hypertension, raised arterial stiffness and vascular remodeling in mice. PLoS One 9: e114908, 2014.

11. Park S, Lee LR, Seo JH and Kang S: Curcumin and tetrahydrocurcumin both prevent osteoarthritis symptoms and decrease the expressions of pro-inflammatory cytokines in estrogen-deficient rats. Genes Nutr 11: 2, 2016.

12. Wu JC, Lai CS, Badmaev V, Nagabhushanam K, Ho CT and Pan MH: Tetrahydrocurcumin, a major metabolite of curcumin, induced autophagic cell death through coordinative modulation of PI3K/Akt-mTOR and MAPK signaling pathways in human leukemia HL-60 cells. Mol Nutr Food Res 55: 1646-1654, 2011.

13. Xiang L, Nakamura Y, Lim YM, Yamasaki Y, Kurokawa-Nose Y, Maruyama W, Osawa T, Matsuura A, Motoyama N and Tsuda L: Tetrahydrocurcumin extends life span and inhibits the oxidative stress response by regulating the FOXO forkhead transcription factor. Aging (Albany NY) 3: 1098-1109, 2011

14. Mukhamedshina YO, Akhmetzyanova ER, Kostennikov AA, Zakirova EY, Galieva LR, Garanina EE, Rogozin AA, Kiassov AP and Rizvanov AA: Adipose-derived mesenchymal stem cell application combined with fibrin matrix promotes structural and functional recovery following spinal cord injury in rats. Front Pharmacol 9: 343, 2018.

15. Sharp KG, Gramer R, Butler L, Cramer SC, Hade E and Page SJ: Effect of overground training augmented by mental practice on gait velocity in chronic, incomplete spinal cord injury. Arch Phys Med Rehabil 95: 615-621, 2014.

16. van der Scheer JW, de Groot S, Tepper M, Faber W; ALLRISC group, Veeger DH and van der Woude LH: Low-intensity wheelchair training in inactive people with long-term spinal cord injury: A randomized controlled trial on fitness, wheelchair skill performance and physical activity levels. J Rehabil Med 48: 33-42, 2016.

17. Shin JC, Kim KN, Yoo J, Kim IS, Yun S, Lee H, Jung K, Hwang K, Kim M, Lee IS, et al: Clinical trial of human fetal brain-derived neural stem/progenitor cell transplantation in patients with traumatic cervical spinal cord injury. Neural Plast 2015: 630932, 2015.

18. Murai T, Murata R, Manabe Y, Sugie C, Tamura T, Ito H, Miyoshi Y and Shibamoto Y: Intensity modulated stereotactic body radiation therapy for single or multiple vertebral metastases with spinal cord compression. Pract Radiat Oncol 4: e231-e237, 2014.
19. Zheng B, Ye L, Zhou Y, Zhu S, Wang Q, Shi H, Chen D, Wei X, Wang Z, Li X, et al: Epidermal growth factor attenuates blood-spinal cord barrier disruption via PI3K/Akt/Rac1 pathway after acute spinal cord injury. J Cell Mol Med 20: 1062-1075, 2016.

20. Miranpuri GS, Schomberg DT, Alrfaei B, King KC, Rynearson B, Wesley VS, Khan N, Obiakor K, Wesley UV and Resnick DK: Role of matrix metalloproteinases 2 in spinal cord injury-induced neuropathic pain. Ann Neurosci 23: 25-32, 2016.

21. Schreiber R, Paim LR, de Rossi G, Matos-Souza JR, Costa E Silva Ade A, Souza CM, Borges M, Azevedo ER, Alonso KC, Gorla JI, et al: Matrix metalloproteinases and left ventricular function and structure in spinal cord injured subjects. Clin Chim Acta 437: 136-140, 2014.

22. Zhang H, Chu G, Pan C, Hu J, Guo C, Liu J, Wang Y and Wu J: A nutrient mixture reduces the expression of matrix metalloproteinases in an animal model of spinal cord injury by modulating matrix metalloproteinase-2 and matrix metalloproteinase-9 promoter activities. Exp Ther Med 8: 1835-1840, 2014.

23. Lee JY, Kim HS, Oh TH and Yune TY: Ethanol extract of Bupleurum falcatum improves functional recovery by inhibiting matrix metalloproteinases-2 and -9 activation and inflammation after spinal cord injury. Exp Neurobiol 19: 146-154, 2010

24. Cirillo G, Colangelo AM, De Luca C, Savarese L, Barillari MR, Alberghina L and Papa M: Modulation of matrix metalloproteinases activity in the ventral horn of the spinal cord re-stores neuroglial synaptic homeostasis and neurotrophic support following peripheral nerve injury. PLoS One 11: e0152750, 2016.

25. Yodkeeree S, Garbisa S and Limtrakul P: Tetrahydrocurcumin inhibits HT1080 cell migration and invasion via downregulation of MMPs and uPA. Acta Pharmacol Sin 29: 853-860, 2008.

26. Sun Z, Yan B, Yu WY, Yao X, Ma X, Sheng G and Ma Q: Vitexin attenuates acute doxorubicin cardiotoxicity in rats via the suppression of oxidative stress, inflammation and apoptosis and the activation of FOXO3a. Exp Ther Med 12: 1879-1884, 2016.

27. Luo L, Lu AM, Wang Y, Hong A, Chen Y, Hu J, Li X and Qin ZH: Chronic resistance training activates autophagy and reduces apoptosis of muscle cells by modulating IGF-1 and its receptors, $\mathrm{Akt} / \mathrm{mTOR}$ and $\mathrm{Akt} / \mathrm{FOXO} 3 \mathrm{a}$ signaling in aged rats. Exp Gerontol 48: 427-436, 2013

28. Yoshihara T, Kobayashi H, Kakigi R, Sugiura T and Naito H: Heat stress-induced phosphorylation of FoxO3a signalling in rat skeletal muscle. Acta Physiol (Oxf) 218: 178-187, 2016.

29. Luan Y, Chen M and Zhou L: MiR-17 targets PTEN and facilitates glial scar formation after spinal cord injuries via the PI3K/Akt/mTOR pathway. Brain Res Bull 128: 68-75, 2016.

30. Chen CH, Sung CS, Huang SY, Feng CW, Hung HC, Yang SN, Chen NF, Tai MH, Wen ZH and Chen WF: The role of the PI3K/Akt/mTOR pathway in glial scar formation following spinal cord injury. Exp Neurol 278: 27-41, 2016. 